\title{
A New Polyacetylene from Vernonia scorpioides (Lam.) Pers. (Asteraceae) and its in vitro Antitumoral Activity
}

\author{
Humberto Buskuhl, ${ }^{a}$ Rilton A. Freitas, ${ }^{a}$ Franco Delle Monache,${ }^{b}$ Andersson Barison, ${ }^{c}$ \\ Francinete R. Campos, ${ }^{c}$ Yuri E. Corilo, ${ }^{d}$ Marcos N. Eberlin ${ }^{d}$ and Maique W. Biavatti ${ }^{*, a, \#}$ \\ ${ }^{a}$ Centro de Ciências da Saúde, Universidade do Vale do Itajaí, 88302-202 Itajaí-SC, Brazil \\ ${ }^{b}$ Dipartimento di Chimica e Tecnologia delle Sostanze Biologicamente Attive, Università "La \\ Sapienza", Roma, Italy \\ 'Departamento de Química, Centro Politécnico, Universidade Federal do Paraná, 81530-900 \\ Curitiba-PR, Brazil \\ ${ }^{d}$ Instituto de Química, Universidade Estadual de Campinas, 13083-970 Campinas-SP, Brazil
}

Vol. 20, No. 7, 1327-1333, 2009.

Page 1331, column 2, line 9, the optical rotation has been included:

5-Octa-2,4,6-triynyl-furan-2(5H)-one (1)

Colorless crystals $\left(\mathrm{CHCl}_{3}\right), \mathrm{mp} 98-100{ }^{\circ} \mathrm{C} ;[\alpha]_{\mathrm{D}}^{24}=+138.3\left(\mathrm{c}=0.3\right.$, dichloromethane); $\mathrm{UV}(\mathrm{MeOH}) \lambda_{\max } / \mathrm{nm}:(\log \in) 207$ (5.58); IR (KBr) $v_{\max } / \mathrm{cm}^{-1}: 3081,2515,2438,1786,1289,791,679,631,551,329 ;{ }^{1} \mathrm{H}$ and ${ }^{13} \mathrm{C}$ NMR data shown in Table 1; ESI-MS $\mathrm{m} / z$ 183.0 [M - H] ${ }^{-}$; ESI-MS/MS (daughter ions, 25\%) $\mathrm{m} / \mathrm{z} 139$ [M - H - $\left.\mathrm{CO}_{2}\right]^{-}$(100), 87 (3); HR-TOF-MS (ESI positive) $m / z 185.0597[\mathrm{M}+\mathrm{H}]^{+}$(calc. for $\mathrm{C}_{12} \mathrm{H}_{8} \mathrm{O}_{2}+\mathrm{H}^{+}$, 185.0603).

*e-mail: maique@ccs.ufsc.br

\# Present address: Laboratório de Farmacognosia, Departamento de Ciências Farmacêuticas, Centro de Ciências da Saúde, Universidade

Federal de Santa Catarina, Trindade, 88040-900 Florianopolis-SC, Brazil 